\title{
TOUSCHEK EFFECT CALCULATION AND ITS APPLICATION TO A TRANSPORT LINE*
}

\author{
A. Xiao ${ }^{\dagger}$, M. Borland, ANL, Argonne, IL 60439, USA
}

\section{Abstract}

The Touschek effect is a major concern for lepton storage rings of low emittance (i.e., high bunch density) and low or moderate beam energy, such as third-generation synchrotron light sources. Piwinski's formula, which includes beam shape variation along the beamline and which is suitable for any beam energy, has been incorporated into a program that interoperates with elegant for use in lifetime calculations. The difference between using Piwinski's method and other simplified methods for the APS is shown in this paper. Furthermore, because of the generality of this formula, we also applied it to transport lines to predict beam loss rates and beam loss locations for the first time. An example related to a possible energy recovery linac upgrade of the APS (APS-ERL) is also given in this paper.

\section{INTRODUCTION}

Coulomb scattering of charged particles traveling together causes an exchange of momentum between the transverse and longitudinal directions. Due to relativistic effects, the momentum transferred from the transverse to the longitudinal direction is enhanced by the factor $\gamma$. For stored beam, particles are lost if their longitudinal momentum deviation exceeds the rf bucket or the momentum aperture determined by the lattice. This is called the Touschek effect and is generally the limiting factor in beam lifetime for third-generation light sources.

An analytical formula for calculating Touschek scattering half-lifetime of a flat beam, with non-relativistic transverse momentum is given by $[1,2]$

$$
\frac{1}{\tau}=\left\langle\frac{r_{0}^{2} c N}{8 \pi \sigma_{x} \sigma_{y} \sigma_{s}} \frac{\sqrt{\xi} D(\xi)}{\gamma^{2} \eta^{3}}\right\rangle,
$$

with

$$
\begin{gathered}
\xi=\left(\frac{\eta \beta_{x}}{\gamma \sigma_{x}}\right)^{2} \\
D(\xi)=-\frac{3}{2} e^{-\xi}+\frac{\xi}{2} \int_{\xi}^{\infty} \frac{\ln u}{u} e^{-u} d u \\
+\frac{3 \xi-\xi \ln \xi+2}{2} \int_{\xi}^{\infty} \frac{e^{-u}}{u} d u
\end{gathered}
$$

where $r_{0}$ is the classical electron radius, $c$ is the speed of light, $N$ is the number of particles inside the bunch, $\sigma_{x, y, s}$

\footnotetext{
${ }^{*}$ Work supported by the U.S. Department of Energy, Office of Science, Office of Basic Energy Sciences, under Contract No. DE-AC02$06 \mathrm{CH} 11357$.

$\dagger$ xiaoam@aps.anl.gov
}

05 Beam Dynamics and Electromagnetic Fields are the beam sizes in each direction, $\gamma$ is the Lorenz factor, and $\eta=\frac{d p}{P_{0}}$ is the momentum acceptance of the ring. This simple formula is used in many computer codes.

Piwinski [3] derived a more general formula (see Equation 4 below), which includes beam envelope variation and is suitable for arbitrary energies. Inspired by F. Wang's [4] work at MIT and taking advantage of elegant [5], which has the ability to calculate the local momentum aperture at each point in a system, we wrote an application code using Piwinski's result. This code has been used for APS beam lifetime calculations and compared with results from Equation 1 .

The APS has been in operation for 11 years. The effective emittance has been decreased from the original value of $8 \mathrm{~nm}$ to $3.1 \mathrm{~nm}$. This value is now limited by existing hardware capabilities. For further improvement, either a new ring or an ERL-based facility is needed. The APS Machine Adversary Committee (MAC) met in 2006 and reached the conclusion that an ERL-based upgrade is the most suitable course for APS [6]. We already know that for APS, the most dominant continuous beam loss mechanism is the Touschek effect. The APS-ERL [7] upgrade would have the same beam energy $(7 \mathrm{GeV})$ and beam current $(100$ $\mathrm{mA}$ ) as APS today, but with much smaller transverse emittance and shorter bunch length. The Touschek scattering effects will thus be much stronger. Several questions need to be answered:

- What will the loss rate be?

- Where will the scattered particles be lost?

- Will the current APS tunnel provide enough radiation shielding?

The APS-ERL is a transport line, so the question of how to simulate off-momentum particle loss due to dispersion and chromatic effects is a new question that needs to be answered. A direct simulation method [8] can be used for solving such problems, but it needs code development and requires substantial computational time for an accurate answer. We can instead apply Piwinski's work to quickly and conveniently answer these questions.

\section{PIWINSKI'S FORMULA}

Piwinski [3] derived a general formula for calculating Touschek lifetime of a stored beam for arbitrary ratios of beam height to beam width and arbitrary energies in the rest frame of the colliding particles. Variations in the beam envelopes are taken into account as well. In a simple form, the lifetime $T_{l}$ can be defined by

$$
\frac{1}{T_{l}}=\left\langle\frac{R}{N_{0}}\right\rangle,
$$


where $N_{0}$ is the number of particles in a bunch, and $R$ is the total number of scattering events per unit time. The average is taken along the beam's closed orbit.

$$
R=\frac{r_{p}^{2} c \beta_{x} \beta_{y} \sigma_{h} N_{p}^{2}}{8 \sqrt{\pi} \beta^{2} \gamma^{4} \sigma_{x \beta}^{2} \sigma_{y \beta}^{2} \sigma_{s} \sigma_{p}} F\left(\tau_{m}, B_{1}, B_{2}\right),
$$

with

$$
\begin{gathered}
B_{1}=\frac{1}{2 \beta^{2} \gamma^{2}}\left[\frac{\beta_{x}^{2}}{\sigma_{x \beta}^{2}}-\frac{\beta_{x}^{2} \sigma_{h}^{2} \tilde{D}_{x}^{2}}{\sigma_{x \beta}^{4}}+\frac{\beta_{y}^{2}}{\sigma_{y \beta}^{2}}-\frac{\beta_{y}^{2} \sigma_{h}^{2} \tilde{D}_{y}^{2}}{\sigma_{y \beta}^{4}}\right] \\
B_{2}^{2}=B_{1}^{2}-\frac{\beta_{x}^{2} \beta_{y}^{2} \sigma_{h}^{2}}{\beta^{4} \gamma^{4} \sigma_{x \beta}^{4} \sigma_{y \beta}^{4} \sigma_{p}^{2}}\left(\sigma_{x}^{2} \sigma_{y}^{2}-\sigma_{p}^{4} D_{x}^{2} D_{y}^{2}\right) \\
\tau_{m}=\beta^{2} \delta_{m}^{2}=\beta^{2}\left(\frac{\Delta p_{m}}{p}\right)^{2}, \\
\sigma_{h}=\frac{\sigma_{x \beta} \sigma_{y \beta} \sigma_{p}}{\sqrt{\tilde{\sigma}_{x}^{2} \sigma_{y \beta}^{2}+\tilde{\sigma}_{y}^{2} \sigma_{x \beta}^{2}-\sigma_{x \beta}^{2} \sigma_{y \beta}^{2}}} \\
\tilde{D}_{x, y}=\alpha_{x, y} D_{x, y}+\beta_{x, y} D_{x, y}^{\prime}, \\
\tilde{\sigma}_{x, y}^{2}=\sigma_{x \beta, y \beta}^{2}+\sigma_{p}^{2}\left(D_{x, y}^{2}+\tilde{D}_{x, y}^{2}\right), \\
F_{=}^{\infty} \int_{\tau_{m}}^{-B_{1} \tau} I_{0}\left(B_{2} \tau\right) \frac{\sqrt{\tau} d \tau}{\sqrt{1+\tau}}\left(\left(2+\frac{1}{\tau}\right)^{2}\left(\frac{\tau / \tau_{m}}{1+\tau}-1\right)\right. \\
\left.+1-\frac{\sqrt{1+\tau}}{\sqrt{\tau / \tau_{m}}}-\frac{4 \tau+1}{2 \tau^{2}} \ln \frac{\tau / \tau_{m}}{1+\tau}\right),
\end{gathered}
$$

where $r_{p}$ is the classical particle radius; $N_{p}$ is number of particles per bunch; $\beta_{x, y}, \alpha_{x, y}, D_{x, y}$, and $D_{x, y}^{\prime}$ are the local optical functions; $\beta$ and $\gamma$ are the Lorentz factors; $\sigma_{p}$ is the beam energy spread; $\sigma_{x, y, s}$ are the beam sizes in each direction; and $\sigma_{x \beta, y \beta}$ are the beam sizes excluding momentum spread.

\section{TOUSCHEK LIFETIME CALCULATION}

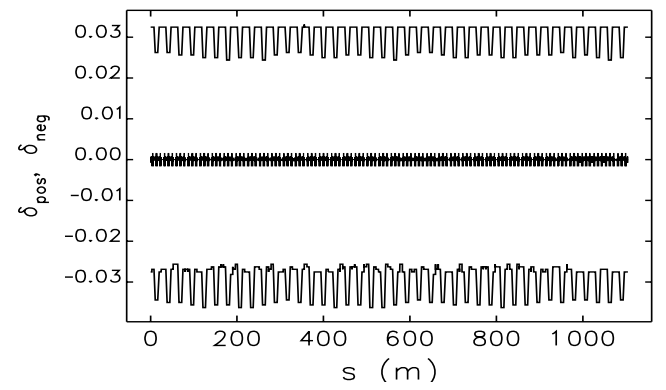

Figure 1: Momentum aperture of the APS.

Equation 4 was implemented in the touschekLifetime software that is distributed with elegant. Here we give an example of applying it to the APS storage ring. The momentum aperture of APS from elegant is shown in Figure 1. The calculated Touschek lifetime is shown in Figure 2, using $\sigma_{s}=12 \mathrm{~mm}$ and $I_{b}=8 \mathrm{~mA}$. The lifetime

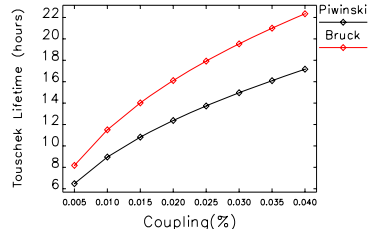

(a)

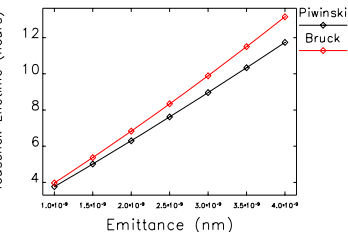

(b)

Figure 2: (a) Touschek lifetime vs. coupling $\left(\epsilon_{x}=3 \mathrm{~nm}\right)$; (b) Touschek lifetime vs. emittance (coupling=1\%).

was calculated for various coupling values for nominal horizontal emittance and as a function of horizontal emittance (assuming it could be varied) using Piwinski's method and Equation 1. Comparing these results to data from APS, where we observe a beam lifetime of 6 - 8 hours with $1 \%$ coupling, we see that Piwinski's method provides more accurate results than the simpler method.

\section{APPLICATION TO APS-ERL}

A possible layout of an APS-ERL upgrade is shown in Figure 3. It mainly consists of the linac, turn-aroundarc (TAA), transport-arc (TA), and the APS. The proposed beam parameters, scaled from Cornell values [9], are given in Table 1. HF, HC, and US stand, respectively, for High Flux, High Coherence and UltraShort mode. Compared to the current APS, the beam energy and total beam current are the same, but we have much smaller transverse beam emittance and bunch length, which will result in stronger Touschek scattering. Since Touschek scattering is the dominant source of particle losses in APS, it's reasonable to expect a significant effect for the ERL. Since the APS tunnel is already built, radiation safety is a concern. To calculate beam loss rates and locations for transport lines like the APS-ERL, we modified Piwinski's formula somewhat. Regardless of whether we have a storage ring or transport

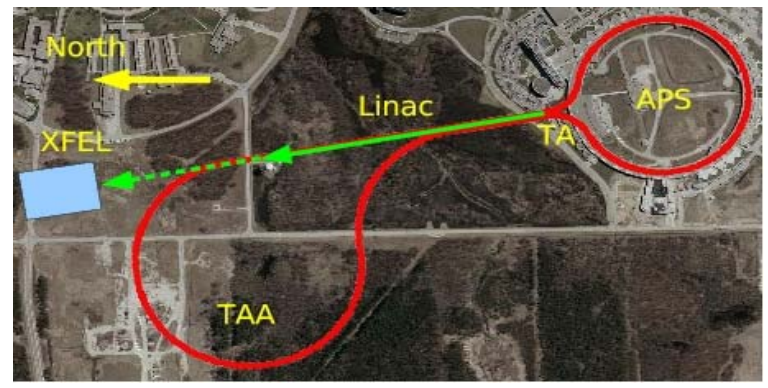

Figure 3: Layout of proposed APS-ERL upgrade.

line, Equation 5 gives, for any location $s$, the scattering rate to energy deviation $\delta>\delta_{m}$ (see Equation 8). When particles travel through a transport line of length $L$ from $s_{1}$ to $s_{2}$, the total scattering rate $W$ for particles having scattered energy deviation $\delta>\delta_{m}$ can be written as:

$$
W(L)_{\delta>\delta_{m}}=\int_{s_{1}}^{s_{2}} R(s) \frac{d s}{V(s)} f_{0},
$$


Table 1: APS-ERL Beam Parameters

\begin{tabular}{|l|c|c|c|c|}
\hline & APS & HF & HC & US \\
\hline Average current (mA) & 100 & 100 & 25 & 1 \\
\hline Repetition rate (MHz) & $0.3-352$ & 1300 & 1300 & 1 \\
\hline Bunch charge (nC) & $0.3-60$ & 0.077 & 0.019 & 1 \\
\hline Emittance (H) (nm) & 3.1 & 0.022 & 0.006 & 0.37 \\
\hline Emittance (V) (nm) & 0.025 & 0.022 & 0.006 & 0.37 \\
\hline Bunch length (ps) & $20-70$ & 2 & 2 & 0.1 \\
\hline Momentum spread (\%) & 0.1 & 0.02 & 0.02 & 0.3 \\
\hline
\end{tabular}

where $R(s)$ is the local scattering rate, $V(s)$ is the local beam velocity, and $f_{0}$ is the bunch repetition rate.

Assuming, for example, that there are momentum apertures at locations $L_{1}$ and $L_{2}$, then the total beam loss rate between $L_{1}$ and $L_{2}$ will be $W\left(L_{2}\right)-W\left(L_{1}\right)$.

Equation 13 gives a very simple way for determining beam loss rate and beam loss location. Applying it to the APS-ERL lattice design, the calculated $W$ at end of APS vs. scattered energy deviation is shown in Figure 4. The loss rate limitation based on the existing shielding is about $170 \mathrm{pA} / 1100 \mathrm{~m}$ (giving $1 \mathrm{mrem} / \mathrm{hour}$ outside the shield wall) [10], which corresponds to $\pm 1 \%$ momentum acceptance. The APS ring now has momentum aperture $\geq 2 \%$, so this seems easily achievable.

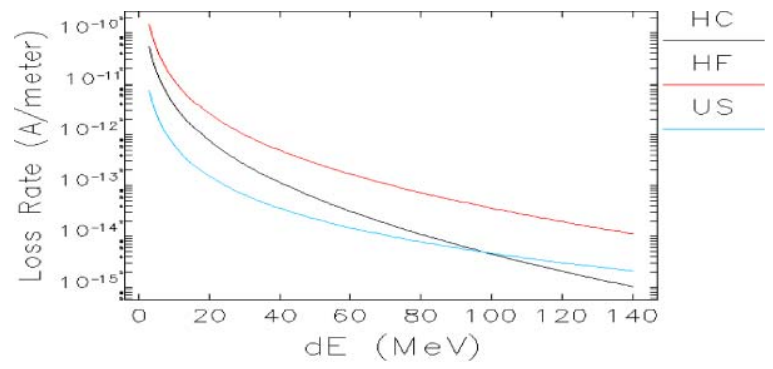

Figure 4: Beam loss rate vs. scattered energy deviation at the end of the APS storage ring.

A detailed tracking study shows that if there are no sextupoles installed in the TAA, the momentum acceptance is very small (see Figure 5(a)), and beam loss due to Touschek scattering is likely to be unacceptable. We added sextupoles to the TAA and performed optimization using tracking with Pelegant [11]. The penalty function included the number of particles lost as well as the deviation of the mean momentum offset of the surviving particles from 0 . We started with a zero-emittance, zeroenergy-spread beam, but included momentum scattering in a uniform $\pm 2 \%$ distribution along the $7-\mathrm{GeV}$ portion of the APS-ERL, allowing each beam particle to be scattered only once (reflecting the single-scattering character of the Touschek effect). The resulting momentum aperture is shown in Figure 5(b). Any beam loss at the beginning of the APS ring can be dealt with by installing a collimator.
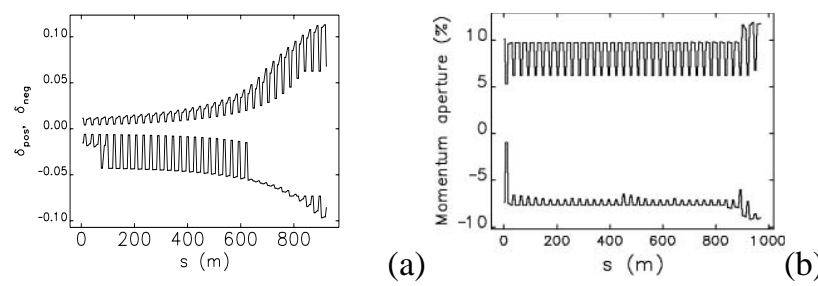

Figure 5: Momentum aperture limits for APS before (a) and after (b) sextupole optimization using Pelegant [11].

\section{CONCLUSION}

Piwinski's Touschek scattering formula has been coded for use with elegant and released as the touschekLifetime program. We compared results for the APS ring and found it can predict beam lifetime more closely matching experiment results. Due to the generality of the formula, it has been used for calculation of the beam loss rate for an APS ERL upgrade option. This simple method can give a quick estimate of the required momentum aperture to keep losses below a defined level. Results show that APS shielding should be adequate provided we optimize sextupoles throughout the system. We are planning to add an accurate Monte Carlo simulation of Touschek scattering to elegant to perform more detailed analysis.

\section{REFERENCES}

[1] J. LeDuff, "Current and Density Limitations in Existing Electron Storage Rings," NIM A239, 83, 1985.

[2] H. Bruck, "Accelerateurs Circulaires de Particules," Presses universitaires de France, Paris, 1966.

[3] A. Piwinski, "The Touschek Effect in Strong Focusing Storage Rings,” DESY 98-179, November 1998.

[4] F. Wang, private conversation.

[5] M. Borland, "elegant: A Flexible SDDS-Compliant Code for Accelerator Simulation," Advanced Photon Source LS-287, September 2000.

[6] http://www.aps.anl.gov/News/Conferences/2006/ APS_Upgrade/index.html

[7] M. Borland, G. Decker, A. Nassiri, Argonne National Laboratory, to be published at PAC07.

[8] S. Khan, "Simulation of the Touschek Effect for BESSY-II: A Monte Carlo Approach," EPAC 94, 1192-1194, June 1994, http://www.jacow.org.

[9] G. Hoffstaetter, FLS 2006 Workshop, DESY, http://www.jacow.org.

[10] "Advanced Photon Source Final Safety Assessment Document, APS-3.1.2.1.0, June 1996.

[11] Y. Wang and M. Borland, ANL, Argonne National Laboratory, to be published at PAC07.

05 Beam Dynamics and Electromagnetic Fields

D03 High Intensity - Incoherent Instabilities, Space Charge, Halos, Cooling 